\title{
Strong Spurious Noise Suppression for an FMCW SAR
}

\author{
Haishan TIAN ${ }^{1}$, Wenge CHANG ${ }^{1}$, Xiangyang $L I^{1}$, Zhaohe LIU ${ }^{2}$ \\ ${ }^{1}$ College of Electronic Science \& Engineering, National University of Defense Technology, Deya Str. 109, Changsha, China \\ ${ }^{2}$ Science and Technology on Near-Surface Detection Laboratory, West Tonghui Road 160, Wuxi, P. R. China \\ haishan_tian@163.com, changwenge@nudt.edu.cn, lxyniu@sina.com, Lzhh@vip.sina.com
}

Manuscript received February 4, 2016

\begin{abstract}
To meet the miniature requirement, a frequency modulated continuous wave synthetic aperture radar (FMCW SAR) puts tight constraint on the compactness, which causes the interference of narrow band noise. The aim of this study is to suppress the strong noise for an FMCW SAR. First, the quantitative analysis of the noise is performed. It is found that a strong spurious noise of the analog-to-digital converter $(A D C)$ is introduced from interferences and significantly affects the image quality; the other noise components are sufficiently small, thus having ignorable influences. Then, a Fast Fourier Transform (FFT) based method of noise suppression is proposed to eliminate the ADC strong spurious noise, adopting an $A D C$ and a field programmable gate array (FPGA). Finally, using the real Ku-band FMCW SAR data, the level of the noise components is measured and the effectiveness of the proposed noise suppression method is validated. The results show that the measured noise level coincides with the theoretical noise level, and the proposed noise suppression method effectively eliminates the ADC strong spurious noise.
\end{abstract}

\section{Keywords}

Frequency Modulated Continuous Wave (FMCW), Synthetic Aperture Radar (SAR), strong spurious noise, noise suppression, miniaturized system

\section{Introduction}

Frequency modulated continuous wave synthetic aperture radar (FMCW SAR) has become popular in the radar field due to its low cost, light weight, small size and high-resolution imagery [1-7]. This type of the SAR provides the observation flexibility required in the cost effective application. The College of Electronic Science and Engineering (CESE) of the National University of Defense Technology (NUDT) built an FMCW SAR in 2014 [8]. The realized FMCW SAR works at Ku-band with the weight of $2.4 \mathrm{~kg}$, the size of $140 \times 140 \times 100 \mathrm{~mm}^{3}$ and the power consumption of $45 \mathrm{~W}$, while it has the ability of real-time imaging [9]. Compared with the existed FMCW SARs [5-7], this system puts more constraints on the com- pactness. Such a miniaturized system may result in a significant increase of the noise level due to the potential interferences and the lack of the precise motion sensors. Therefore, the aim of this study is to suppress the strong noise of the miniature FMCW SAR.

Based on the characteristic of the noise for the SAR, the additive and multiplicative noises are identified. The receiver thermal noise is one of the principal contributors to the additive noise and is introduced in [10]. The noise of the analog-to-digital converter (ADC) is a potential principal contributor to the additive noise, because the narrowband interferences (NBI) may be introduced into the ADC. Many methods have been proposed to eliminate the interferences from the interference-corrupted signal. The rejection methods can be classified as those based upon adaptive filtering and those based upon transform domain processing structures. The adaptive filters, such as least mean square estimation (LMS) adaptive filters [11-13] and lattice adaptive filters [14], usually adopt iterative algorithms to reduce convergence time. The iterative algorithm relates to many operations, such as multiplications and trigonometry operations. However, these operations are not very suitable to be performed in some devices like the field programmable gate array (FPGA) [15]. The other type of rejection method is to process the received signal by surface acoustic wave (SAW) [16] technology or Fast Fourier Transform (FFT) and then estimate the interference from sharply peaked spectrum. The processing of this method is easily associated with the subsequent imaging of FMCW SAR. However, it is difficult to obtain the interference when the signal at the frequency of interference can be comparable to the interference. $\mathrm{Xu}$ et al. proposed the wavelet-based removal of sinusoidal interference from a signal [17] without notable attenuation to the signal at interference frequencies. However, the significant distortion to signal will be caused when the SNR is low and when there is a sharp transition for this method, for example, when the FMCW SAR passes through riverside. With the multiplicative noise, its principal contributors are the integrated sidelobes noise, the ambiguity noise, and the quantization noise. Zhao et al. [18] proposed a compensation method for the phase errors of an FMCW SAR. However, the method is of limited effect on the high frequency phase errors which are one of the principal contributors to the sidelobes noise. The ambiguity noises has the marginal 
influence on the imagery of the airborne FMCW SAR system because of its short slant range and high pulse repetition frequency (PRF) [19-22]. Therefore, the ambiguity noise is not considered in this study. Similarly, the quantization noise is sufficiently small [10] and is not considered as well.

In this study, a Ku-band FMCW SAR developed by NUDT is taken as an example to analyze the noise level of the system. First, a quantitative analysis of the noise components is performed. Then, a FFT based method is proposed to eliminate the strong noise adopting an ADC and a FPGA. Finally, using the real FMCW SAR data, the levels of the noise components are measured, and the effectiveness of the proposed noise suppression method is validated.

The remainder of the study is organized as follows. In Sec. 2, a brief introduction of an FMCW SAR system and its geometry model is presented. In Sec. 3, the quantitative analysis of the noise components is performed. In Sec. 4, the method of strong noise suppression is proposed using an ADC and a FPGA. In Sec. 5, the noises levels are measured, and the effectiveness of noise suppression are validated using the real FMCW SAR data. Finally, conclusions are drawn in Sec. 6.

\section{System Introduction and its Geometry Model}

The diagram of FMCW SAR system is illustrated in Fig. 1, where FMCW signal is generated and amplified, then fed to transmitting antenna. The backscattered echo is received by the receiving antenna, magnified by the amplifier, and mixed with the transmitting signal coupled from the transmitter. Then the backscattered echo turns to be a narrow bandwidth signal which is corresponding to the observed swath in range using the dechirp method. After sampled by an ADC, the dechirped signal is processed to generate the final imaging data in real time in the digital processing module.

The geometry model depicted in Fig. 2 is used to show the model of FMCW SAR. The airplane moves along $\mathrm{X}$ axis with a velocity of $v$, and $t_{m}$ stands for the time variable in azimuth. $H$ denotes the height of the platform. $T_{0}$ stands for a point scatter with a three-dimensional coordinate of $\left(x_{0}, z_{0}, 0\right)$. $R$ and $R$ ' are the closest slant range and

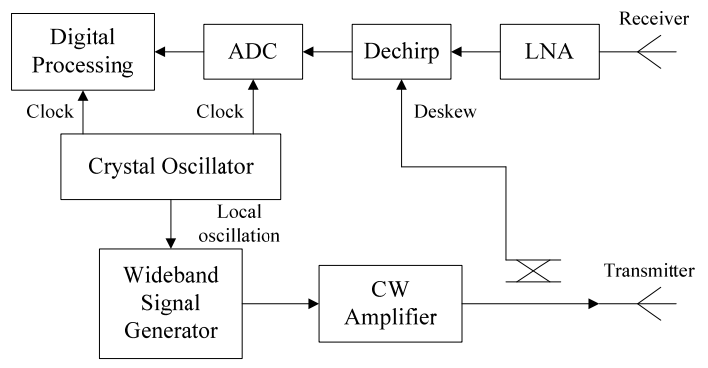

Fig. 1. The diagram of FMCW SAR system.

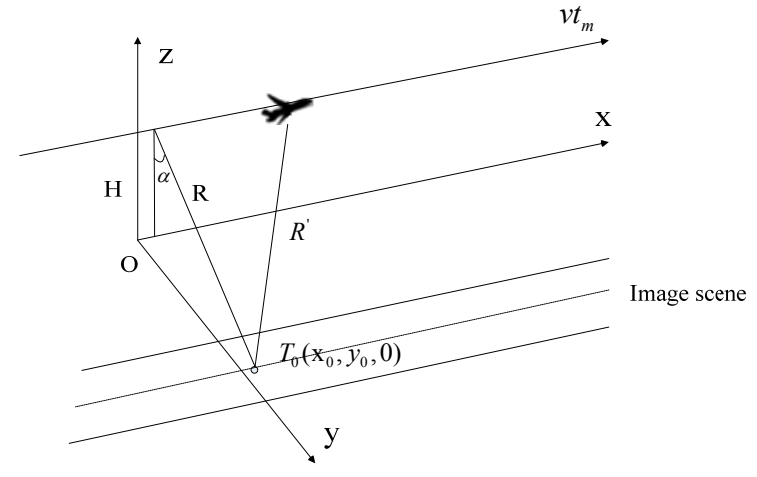

Fig. 2. FMCW SAR system geometry in an ideal case.

\begin{tabular}{|l|c|}
\hline \multicolumn{1}{|c|}{ Parameter } & Value \\
\hline LFM bandwidth & $600 \mathrm{MHz}$ \\
Receiver noise figure & $3 \mathrm{~dB}$ \\
Low noise amplification & $60 \mathrm{~dB}$ \\
Dechirped signal frequency & $6-14 \mathrm{MHz}$ \\
Range and azimuth resolution & $0.35 \mathrm{~m} \times 0.35 \mathrm{~m}$ \\
ADC sampling frequency & $100 \mathrm{MHz}$ \\
\hline
\end{tabular}

Tab. 1. Parameters of an FMCW SAR system.

instantaneous slant range from the radar to the point $T_{0}$. $\alpha$ is the pitching angle, and $\sin \alpha=\sqrt{1-(H / R)^{2}}$.

A part of parameters of an FMCW SAR system are listed in Tab. 1.

\section{Noise Analysis}

The total noise of an FMCW SAR system includes additive noise and multiplicative noise. It is expressed by

$$
\sigma_{\mathrm{N}}=\sigma_{\mathrm{n}}+(M N R) \bar{\sigma}_{0}
$$

where $\sigma_{\mathrm{n}}$ is the effective backscatter coefficient of the additive noise; $\overline{\sigma_{0}}$ is the average backscatter coefficient of the scene; $M N R$ is the multiplicative noise ratio which is the constant of proportionality between the multiplicative noise and the average signal level [23].

\subsection{Additive Noise}

In an FMCW SAR, the main contributors to the additive noise are the receiver thermal noise and the ADC spurious noise. Thise additive noise is characterized by $\sigma_{\mathrm{n}}$, which is the equivalent terrain radar backscatter coefficient that would produce a signal equal to the noise.

\subsubsection{Receiver Thermal Noise}

The signal-to-noise ratio $(S N R)$ of the imagery [10] is

$$
S N R_{\text {clutter }}=\frac{P_{\mathrm{t}} G_{\mathrm{t}} G_{\mathrm{r}}}{2 N_{0} L}\left(\frac{\lambda}{4 \pi R}\right)^{3}\left[\frac{\rho_{\mathrm{r}} \sigma_{0}}{K_{\mathrm{r}} v \sin \alpha}\right]
$$

where $P_{\mathrm{t}}$ is the transmitted power; $G_{\mathrm{t}}$ and $G_{\mathrm{r}}$ are the transmit and receive antenna gains, respectively; $L$ is the loss factor; $\lambda$ is the center transmitted wavelength; $R$ is the slant range of the scatterer; $K_{\mathrm{r}}$ is the mainlobe broadening factor 
in range; $\rho_{\mathrm{r}}$ is the range resolution; $\sigma_{0}$ is the backscatter coefficient of the scatterer; $N_{0}$ is the power spectral density $(P S D)$ of the receiver thermal noise, which is

$$
N_{0}=k T_{0} F_{\mathrm{n}}
$$

where $k$ is the Boltzmann's constant which equals $1.38 \times 10^{-23} \mathrm{~J} / \mathrm{K} ; T_{0}$ is the standard temperature, $F_{\mathrm{n}}$ is the receiver noise figure which equals $3 \mathrm{~dB}$. Assuming that $T_{0}$ is $290 \mathrm{~K}$, these parameters are submitted into (3). $N_{0}$ equals $8.00 \times 10^{-21} \mathrm{~W} / \mathrm{Hz}$.

Based on the definition of $\sigma_{\mathrm{n}}$, the effective backscatter coefficient of the receiver thermal noise, which is denoted by $\sigma_{\text {receiver }}$, is equal to $\sigma_{0}$ when $S N R_{\text {clutter }}$ is 1 . So $\sigma_{\text {receiver }}$ is

$$
\sigma_{\text {receiver }}=\left[\frac{K_{\mathrm{r}} \nu \sin \alpha}{\rho_{\mathrm{r}}}\right]\left(\frac{2 N_{0} L}{P_{\mathrm{t}} G_{\mathrm{t}} G_{\mathrm{r}}}\right)\left(\frac{4 \pi R}{\lambda}\right)^{3}=\kappa N_{0} \sin \alpha R^{3}(4)
$$

where $\kappa=\left[\frac{2 K_{\mathrm{r}} \nu L}{\rho_{\mathrm{r}} P_{\mathrm{t}} G_{\mathrm{t}} G_{\mathrm{r}}}\right]\left(\frac{4 \pi}{\lambda}\right)^{3}$. Providing that $\kappa$ is equal to $2.10 \times 10^{7}$, the value of $\sigma_{\text {receiver }}$ is

$$
\sigma_{\text {receiver }}=2.10 \times 10^{7} N_{0} \sin \alpha R^{3}=1.68 \times 10^{-13} \sqrt{1-(H / R)^{2}} R^{3} .
$$

\subsubsection{ADC Spurious Noise}

There are two types of spurious noise: two single-frequency strong signals with the frequencies that drift slowly over time, and a weak signal that is uniformly distributed in the frequency band of the dechirped signal. The influence of the spurious noises on the resulting imagery is now analyzed in detail.

First, the ADC spurious noises are measured. To eliminate the influence of the receiver thermal noise on the test results, the ADC input is from an external source (model number AWG70000), and other operations are the same as those in working order of the FMCW SAR. The full scale of the $\mathrm{ADC}$ is $1 \mathrm{~V}$ which is normalized to $0 \mathrm{~dB}$ in Fig. 3. Figure 3(a) denotes the spectrum of the ADC output signal when there is no signal in the input of the ADC. It is shown that there are two strong signals. The strongest one has the frequency of $48.70 \mathrm{MHz}$. The frequency of this signal drifts slowly with time and ranges from 40 to $50 \mathrm{MHz}$, which is not in the frequency band of the dechirped signal. Therefore, this strong signal will be eliminated by the filter in the image processing. With respect to the second strongest signal, it has the amplitude of $-58 \mathrm{~dB}$ and the frequency of $8.59 \mathrm{MHz}$ which is in the frequency band of the dechirped signal. It is called the ADC strong spurious noise. Because this noise may be in the frequency band of the dechirped signal, it is primarily considered in this study. Figure 3(b) denotes the spectrum of the ADC output signal when the input of the ADC is a single-frequency signal. It is shown that the two strong signals except for the input signal have the same amplitudes as those in Fig. 3(a). Their frequencies have the same characteristic as well. Figure 3(c) denotes the frequency of the ADC strong noise versus the time in a test. It is shown

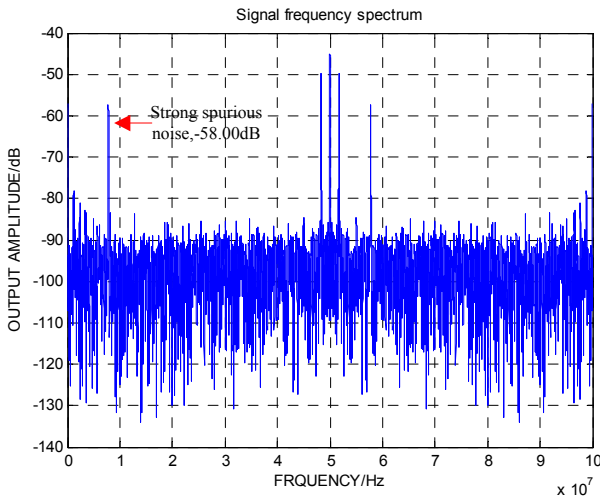

(a)

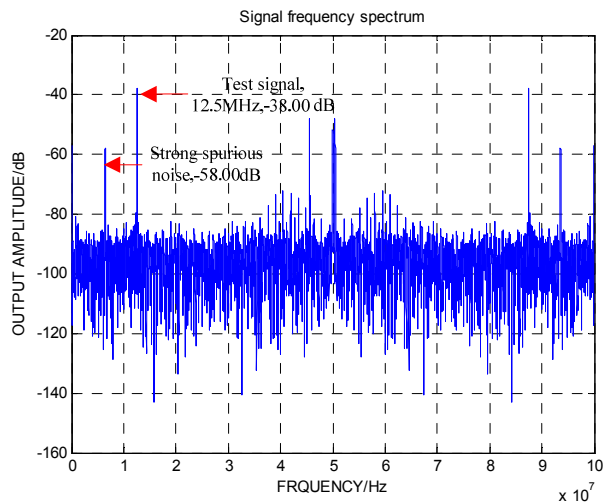

(b)

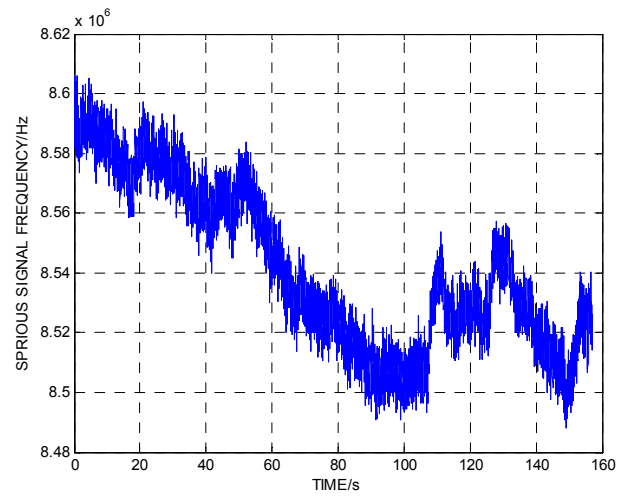

(c)

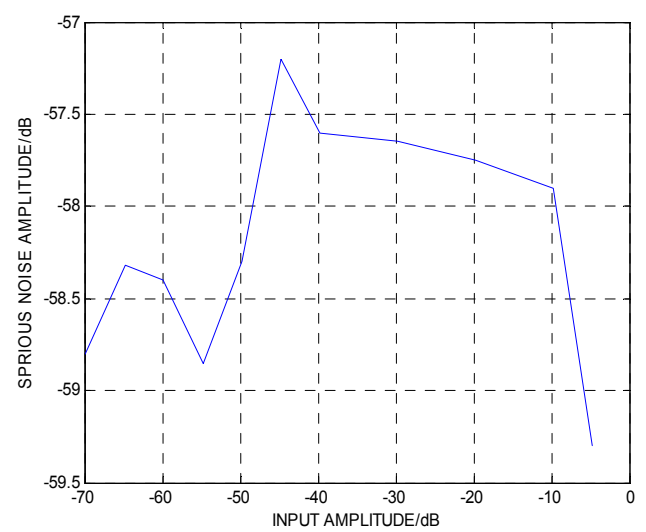

(d)

Fig. 3. (a) Spectrum without an input signal; (b) Spectrum with a single-frequency input signal; (c) Frequency variation; (d) Amplitude variation. 
that the frequency drifts slowly over time. Figure 3(d) describes the amplitude of the ADC strong spurious noise versus the amplitude of the single-frequency input signal. It is shown that the change of the amplitude is sufficiently small. Therefore, the ADC strong spurious noise is independent of the input signal and can be regarded as the additive noise.

Based on the forenamed analysis, the ADC strong spurious noise can be modeled as

$$
n_{\mathrm{s}}(t)=C_{\mathrm{sp}} \exp \left\{-j 2 \pi\left[f(t) t+\theta_{0}\right]\right\}
$$

where $C_{\mathrm{sp}}$ is the amplitude which approximately equals $\left(10^{-58 / 20} \times 1 / 2\right) \mathrm{V}$, and $\theta_{0}$ is the initial phase; $f(t)$ is the frequency, which will vary in the image processing. Because the frequency variation is not the focus in this study, it is not analyzed in depth.

The ADC strong spurious noise is an additive noise. So equation (4) can be also used to calculate the effective backscatter coefficient of the ADC strong spurious noise. That is, the PSD of the receiver thermal noise will be changed to that of the ADC spurious noise when calculating its effective backscatter coefficient in (4).

Because the ADC strong spurious noise is a singlefrequency signal, its power is primarily distributed in the frequency bandwidth of one range resolution cell. The power of the strong spurious noise is

$$
P_{\mathrm{sp}}=\frac{C_{\mathrm{sp}}{ }^{2}}{2 \times 50}=3.96 \times 10^{-9} \mathrm{~W} .
$$

The frequency of the dechirped signal is [10]

$$
f=2 R \gamma / c
$$

where $\gamma$ is the chirp rate of the transmitted signal. Therefore, the frequency bandwidth of the dechirped signal in a range resolution cell is

$$
B_{\mathrm{re}}=2 \rho_{\mathrm{r}} \gamma / c
$$

where $\rho_{\mathrm{r}}$ is the range resolution.

Moreover, $N_{0}$ is the PSD prior to the low noise amplification. The factor of low noise amplification must be considered when calculating the PSD of the ADC strong spurious noise. Therefore, the average PSD of the ADC strong spurious noise distributed in $B_{\mathrm{re}}$ is

$$
P S D_{\mathrm{sp}}=\frac{P_{\mathrm{sp}}}{B_{\mathrm{re}} \cdot \text { Receiver_gain }}=\frac{P_{\mathrm{sp}} \cdot c}{2 \rho_{\mathrm{r}} \gamma \cdot \text { Receiver_gain }}
$$

where Receiver_gain is the low noise amplification. Receiver_gain is $60 \mathrm{~dB}, \rho_{\mathrm{r}}$ equals $0.35 \mathrm{~m}, c$ is $3 \times 10^{8} \mathrm{~m} / \mathrm{s}$ and $\gamma$ is $1.5 \times 10^{12} \mathrm{~Hz} / \mathrm{s}$. These parameters can be substituted into (10), $P S D_{\mathrm{sp}}$ equals $1.13 \times 10^{-18} \mathrm{~W} / \mathrm{Hz} . N_{0}$ is changed to $P S D_{\text {sp }}$ in (4) when calculating the effective backscatter coefficient of ADC strong spurious noise, which is

$$
\sigma_{\mathrm{sp}}=\kappa P S D_{\mathrm{sp}} \sin \alpha R^{3}=2.37 \times 10^{-11} \sqrt{1-(H / R)^{2}} R^{3} .
$$

For the second type of the spurious noise, its power, denoted by $P_{\text {sp_r }}$, can be calculated from the spectrum shown in Fig. 3 (a) and the result is $-36.66 \mathrm{dBmW}$. So its corresponding $P S D$ is

$$
P S D_{\text {sp_r }}=\frac{P_{\text {sp } \mathrm{r}}}{f_{\mathrm{s}} \times \text { Receiver_gain }}=1.30 \times 10^{-22} \mathrm{~W} / \mathrm{Hz}
$$

where $f_{\mathrm{s}}$ is the $\mathrm{ADC}$ sampling frequency which is $100 \mathrm{MHz} . N_{0}$ is changed to $P_{\mathrm{sp} \_\mathrm{r}}$ in (4) when calculating the effective backscatter coefficient of this type of the spurious noise. The result is

$$
\sigma_{\text {sp } \_ \text {r }}=\kappa P S D_{\text {sp } \_ \text {r }} \sin \alpha R^{3}=2.73 \times 10^{-15} \sqrt{1-(H / R)^{2}} R^{3} .
$$

Because $\sigma_{\text {receiver }} / \sigma_{\mathrm{sp} \mathrm{r}}=17.89 \mathrm{~dB}$, the uniformly distributed additive noise thus primarily originates from the receiver thermal noise.

\subsection{Multiplicative Noise}

The principal contributors to the multiplicative noise are the integrated sidelobes noise, the ambiguity noise, and the quantization noise [10]. So the expression of $M N R$ is

$$
M N R=I S L R+Q N R+A M B R
$$

where $I S L R$ is the ratio of all energy in the sidelobes of system impulse response to the energy in the mainlobe, $Q N R$ represents the constant of proportionality between the quantization noise level and the average signal level, and $A M B R$ is the ratio of the ambiguity noise level to the average signal level [10].

The quantization noise is distributed in the entire scene, and its principal contributor to $Q N R$ in an FMCW SAR is the error of the floating-point calculation. The QNR is approximately $-60 \mathrm{~dB}[10]$. With the ambiguity noises, it has the marginal influence on the imagery of the airborne FMCW SAR system [8]. Therefore, the ambiguity noises are not considered in this study.

The integrated sidelobes noise has a masking effect where the signal sidelobes of the echoes from bright scatterers may mask the main response from a weak target [10]. The $I S L R$ includes the range and azimuth $I S L R$, which are denoted by $I S L R_{\mathrm{r}}$ and $I S L R_{\mathrm{a}}$, respectively. They primarily originate from the aperture effect, the high frequency phase errors, and the wideband amplitude errors [10].

The finite data processing aperture is one of the principal contributors to the sidelobe levels. A Fourier transform of the uniformly weighted aperture is $\sin (\pi f) /(\pi f)$ or the sinc function with an ISLR of $-9.7 \mathrm{~dB}$ [10]. An FMCW SAR uses the common Hamming window of which $I S L R_{\mathrm{r}}$ and $I S L R_{\mathrm{a}}$ are both $-35.20 \mathrm{~dB}$.

The high frequency phase errors, which results in the increase of $I S L R_{\mathrm{r}}$, are primarily derived from the high frequency phase error of the generated wideband signal in an FMCW SAR. In Fig. 4(a), the waveform of the generated signal is recorded by a single-shot acquisition of 


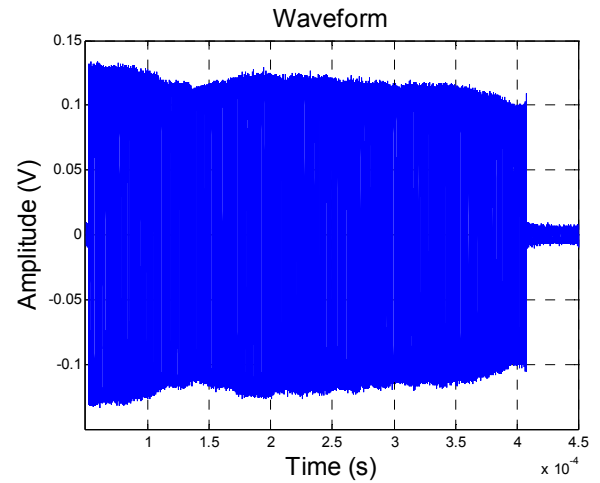

(a)

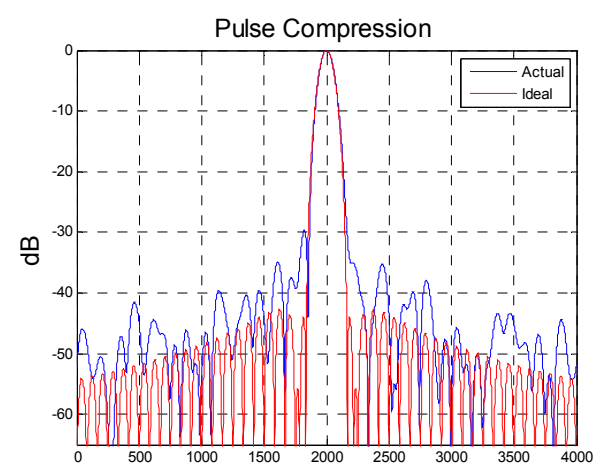

(b)

Fig. 4. (a) Signal waveform; (b) Pulse compression result [23].

a 50 GSPS real-time oscilloscope (model number DPO71254). The pulse compression result of the recorded waveform is shown in Fig. 4(b). The ratio of the energy in the sidelobes to the energy in the mainlobe is approximately equal to $-17.30 \mathrm{~dB}$.

The high-frequency phase errors that result in the increase of $I S L R_{\mathrm{a}}$ are primarily derived from the motion measurement errors of higher order, that is, those varying or changing direction many times over the azimuth aperture [10]. Because the FMCW SAR in this study is a costeffective miniaturized system, the precision of the motion sensors is not sufficiently high to keep motion errors low. To reduce these motion errors, a higher order autofocus technique, i.e., the phase gradient algorithm (PGA) [23], is used in the image processing. Because the motion compensation algorithm is not the focus of this study, its in-depth analysis is not presented in this study.

The principal contributors to the wideband amplitude errors are the radar system's hardware components. The ISLR that is derived from this form of error is significantly lower than that from the phase errors in an FMCW SAR.

\section{Noise Suppression}

Based on the quantitative analysis of the noise in Sec. 3, and assuming that the platform height is $500 \mathrm{~m}$, the effective backscatter coefficients of the receiver thermal noise and the ADC strong spurious noise are calculated using (5) and (12), respectively. Their results are listed in

\begin{tabular}{|c|c|}
\hline Noise component & Theoretical result \\
\hline Receiver thermal noise & $-46.98 \sim-33.66 \mathrm{~dB}$ \\
\hline ADC strong spurious noise & $-25.49 \sim-12.18 \mathrm{~dB}$ \\
\hline $\boldsymbol{I S L} \boldsymbol{R}_{\mathbf{r}}$ & $-17.23 \mathrm{~dB}$ \\
\hline
\end{tabular}

Tab. 2. Theoretical results of the noise components.

the first two items of Tab. 2, where the maximum and minimum values correspond to the farthest and nearest slant range, respectively. The receiver thermal noise level is shown to be small and only comparable to the reflection of the low return terrain [10], such as a quiet body of water. However, the effective backscatter coefficient of the ADC strong spurious noise is shown to be large and can be comparable to that of some targets of interest to an FMCW SAR, such as a road, river beach or cropland. Therefore, this type of noise has considerable influence on the image quality. With the multiplicative noise components, $A M B R$ and $Q N R$ of the airborne FMCW SAR are sufficiently small, which only have a marginal influence on the image quality. $I S L R_{\mathrm{r}}$ is primarily introduced from the high-frequency phase error of the transmitted signal, which is not easily to be extracted and eliminated. $I S L R_{\mathrm{r}}$ equals $10 \log _{10}\left(10^{-35.2 / 10}+10^{-17.3 / 10}\right)=-17.23 \mathrm{~dB}$, as shown in the last item of Tab. 2. The value of $I S L R_{\mathrm{r}}$ does not reach the level which will produce serious deterioration in the image quality. Considering $I S L R_{\mathrm{a}}$, difficulty in sensing the highfrequency phase errors requires a better motion compensation algorithm. According to the quantitative analysis of the noise, it is found that all the noise components except the ADC strong spurious noise are sufficiently small to have marginal or limited influence on the image quality. Therefore, the suppression of the ADC strong spurious noise is investigated in this study.

\subsection{Strong Spurious Noise Suppression}

The suppression of the ADC strong spurious noise is performed based on a monolithic, dual-channel ADC AD9648 and a FPGA XC6VLX75T in this study. The two channels of ADC, which are denoted by channel A and B, are configured as the same operation mode. Their front-end devices, including the operational amplifiers, resistors, and capacitors, have the same models and parameter values. Moreover, their printed circuit board (PCB) layouts are similar as well. When an FMCW SAR works, the two channels simultaneously receive and sample the dechirped signal. Based on (6), the strong spurious noises of channel $A$ and $B$ are expressed by

$$
\begin{aligned}
& n_{\mathrm{A}}\left(n T_{\mathrm{s}}\right)=C_{\mathrm{sp}} \cos \left(2 \pi\left(f_{\mathrm{A}}\left(n T_{\mathrm{s}}\right) n T_{\mathrm{s}}+\varphi_{\mathrm{A}}\right)\right), \\
& n_{\mathrm{B}}\left(n T_{\mathrm{s}}\right)=C_{s \mathrm{p}} \cos \left(2 \pi\left(f_{\mathrm{B}}\left(n T_{\mathrm{s}}\right) n T_{\mathrm{s}}+\varphi_{\mathrm{B}}\right)\right)
\end{aligned}
$$

where $T_{\mathrm{s}}$ is the sampling period, and $f_{\mathrm{A}}\left(n T_{\mathrm{s}}\right)$ and $f_{\mathrm{B}}\left(n T_{\mathrm{s}}\right)$ are the strong spurious noise's frequency of channel A and B, respectively. $\varphi_{\mathrm{A}}$ and $\varphi_{\mathrm{B}}$ are their initial phases. For simplicity, we drop $T_{\mathrm{s}}$ in the following sections. The characteristic of $f_{\mathrm{A}}(n)$ and $f_{\mathrm{B}}(n)$ is as described in Sec. 3.1.2, whereas, their values are different. 
The signal is sampled by two channels of $\mathrm{ADC}$ at the positive and negative edges of the clock, respectively. The outputs of channel A and B are expressed by

$$
\begin{gathered}
s_{\mathrm{A}}(n)=s(n)+n_{\mathrm{A}}(n)+n_{\mathrm{A}_{-} \mathrm{r}}(n), \\
s_{\mathrm{B}}(n)=s(n)+n_{\mathrm{B}}(n)+n_{\mathrm{B}_{-} \mathrm{r}}(n)
\end{gathered}
$$

where $s(n)$ is the sampled data of the ideal signal. $n_{\mathrm{A}_{\mathrm{r}}}(n)$ is the noise of channel A except $n_{\mathrm{A}}(n)$, and $n_{\mathrm{B}_{-}}(n)$ is the noise of channel B except $n_{\mathrm{B}}(n)$.

Because the sampling frequency is $100 \mathrm{MHz}$, the sampling time interval between the two channels is only $5 \mathrm{~ns}$. Therefore, the sampled dechirped signals of the two channels are similar. Based on this consideration, the method for the elimination of the strong spurious noise is proposed. The block diagram of the noise suppression is shown in Fig. 5. The FPGA is the kernel for implementing strong spurious noise suppression.

The noise suppression is divided into four series modules, as shown in Fig. 5. In the module 1, the FFT of the two channels' output signals is firstly performed. Two RAMs before the FFT operation are used because of the difference between the sampling frequency and the clock frequency of the FFT operation. Then the spectrum of channel A's output subtracts that of channel B's output yielding

$$
\begin{aligned}
N_{\mathrm{s}}(k) & =S_{\mathrm{A}}(k)-S_{\mathrm{B}}(k) \\
& =S(k)+N_{\mathrm{A}}(k)+N_{\mathrm{A}_{\mathrm{r}}}(k)-S(k)-N_{\mathrm{B}}(k)-N_{\mathrm{B}_{-} \mathrm{r}}(k) \\
& =N_{\mathrm{A}}(k)+N_{\mathrm{A}_{\mathrm{r}}}(k)-N_{\mathrm{B}}(k)-N_{\mathrm{B}_{-} \mathrm{r}}(k)
\end{aligned}
$$

where $S_{\mathrm{A}}(k), S_{\mathrm{B}}(k), N_{\mathrm{A}}(k), N_{\mathrm{B}}(k), N_{\mathrm{Ar}}(k), N_{\mathrm{Br}}(k)$, and $S(k)$ are the spectrums of $s_{\mathrm{A}}(n), s_{\mathrm{B}}(n), n_{\mathrm{A}}(n), n_{\mathrm{B}}(n), n_{\mathrm{A}_{-} \mathrm{r}}(n)$, $n_{\mathrm{B}} \mathrm{r}(n)$, and $s(k)$, respectively. $k=1,2, \ldots, N$, and $N$ is the data size of FFT operation.

Then, the frequency of channel A's strong spurious noise is extracted in the module 2 . The detailed flow is shown in Fig. 6. The amplitude of $N_{\mathrm{s}}(k)$ i.e., $\left|N_{\mathrm{s}}(k)\right|$ is calculated using the CORDIC IP core [25]. In $\left|N_{\mathrm{s}}(k)\right|$, the frequencies at which the amplitudes of $N_{\mathrm{A}}(k)$ and $N_{\mathrm{B}}(k)$ equal their maximum value are $f_{\mathrm{A}}$ and $f_{\mathrm{B}}$, respectively. Because the amplitudes of $N_{\mathrm{A}}(k)$ and $N_{\mathrm{B}}(k)$ at $f_{\mathrm{A}}$ and $f_{\mathrm{B}}$ are much larger than those of other noise components at the two frequencies. Therefore, $\left|N_{\mathrm{s}}(k)\right|$ has two sharply peaked spectrums at $f_{\mathrm{A}}$ and $f_{\mathrm{B}}$. Moreover, it is known from Section 3.1.2 that the amplitude of ADC spurious noise is time invariant. $f_{\mathrm{A}}$ and $f_{\mathrm{B}}$ are the frequencies, where the value of $\left|N_{\mathrm{s}}(k)\right|$ equals the threshold value corresponding to the $-58 \mathrm{~dB}$ spurious noise. So $f_{\mathrm{A}}$ and $f_{\mathrm{B}}$ can be easily extracted by comparing the value of $\left|N_{\mathrm{s}}(k)\right|$ with the threshold value.

After obtaining $f_{\mathrm{A}}$ and $f_{\mathrm{B}}$, the signal processor does not know which frequency belongs to channel $\mathrm{A}$ at the moment. A frequency judgment is made based on the regularity that the frequency of the ADC strong spurious noise drifts slowly and continuously. Therefore, a frequency being approximate to $f_{\mathrm{A}}$ in the last measurement belongs to channel A. To obtain the initial value of $f_{\mathrm{A}}$, denoted by $f_{\mathrm{A} 0}$, the input of module 2 is changed to the FFT result of the channel A's noise, i.e., no signal in the input of channel A, and output is the desired frequency, as shown in Fig. 6. This is done before the FMCW SAR starts to receive signal.

In module $3, N_{\mathrm{s}}(k)$ is band-pass filtered using a rectangle window to obtain the approximation of $N_{\mathrm{A}}(k)$, denoted by $\hat{N}_{\mathrm{A}}(k)$. That is,

$$
\hat{N}_{\mathrm{A}}(k)=\left\{\begin{array}{cc}
N_{\mathrm{s}}(k) & k=N f_{\mathrm{A}} / f_{\mathrm{s}} \\
0 & \text { else }
\end{array} .\right.
$$

A RAM, denoted RAM3, is to buffer $N_{\mathrm{s}}(k)$ during the operation of module 2 .

Then in module $4, \hat{N}_{\mathrm{A}}(k)$ is subtracted from $S_{\mathrm{A}}(k)$ which yields the desired signal

$$
\begin{aligned}
\hat{S}_{\mathrm{A} 0}(k) & =S_{\mathrm{A}}(k)-\hat{N}_{\mathrm{A}}(k) \\
& = \begin{cases}S(k)+N_{\mathrm{B}}(k)+N_{\mathrm{B}_{\mathbf{r}} \mathrm{r}}(k) & k=N f_{\mathrm{A}} / f_{\mathrm{s}} \\
S(k)+N_{\mathrm{A}}(k)+N_{\mathrm{A}_{\mathbf{r}} \mathrm{r}} & \text { else }\end{cases} \\
& \approx \begin{cases}S(k)+N_{\mathrm{B}_{\mathbf{r}}}(k) & k=N f_{\mathrm{A}} / f_{\mathrm{s}} \\
S(k)+N_{\mathrm{A}_{-} \mathrm{r}}(k) & \text { else }\end{cases}
\end{aligned}
$$

The IFFT of $\hat{S}_{\mathrm{A} 0}(k)$ is performed to obtain the dechirped signal without the strong spurious noise. Because $N_{\mathrm{Ar}}(k)$ and $N_{\mathrm{Br}}(k)$ are all uniformly distributed noises, and have the same levels, the result is expressed by

$$
s_{\mathrm{Ao}}(n) \approx s(n)+n_{\mathrm{A}_{-} \mathrm{r}}(n) .
$$

A RAM, denoted RAM4, is to buffer $S_{\mathrm{A}}(k)$ during the operation of module 2 and module 3 . It can be seen from (22) that the strong spurious noise of channel A i.e., $n_{\mathrm{A}}(n)$, is eliminated. At last, $s_{\mathrm{Ao}}(n)$ is transferred to the DSP for real-time imaging.

For the noise suppression of the FMCW SAR, the most concern is about the suppression level at the frequency of the strong spurious noise. It can be obtained from (21) that the suppression level at $f_{\mathrm{A}}$ is $\left|N_{\mathrm{B}}(k)\right| /\left|N_{\mathrm{A}}(k)\right|$, where $k=N f_{\mathrm{A}} / f_{\mathrm{s}}$. At $f_{\mathrm{A}},\left|N_{\mathrm{B}}(k)\right|$ is sufficiently small in comparison with $\left|N_{\mathrm{A}}(k)\right|$. Therefore, the noise suppression level of this method should be very high.

Then the speed, resource occupancy and precision of the noise suppression are analyzed. All the operations of noise suppression shown in Fig. 5 are performed in the pipelining way. The most time-consuming is the FFT operation with $N$ of $4 \mathrm{~K}$. The measured time consumption of the FFT operation for $4 \mathrm{~K}$ data is $33.23 \mu$ s with the clock frequency of $250 \mathrm{MHz}$, which is shorter than the time for $4 \mathrm{~K}$ data generation, i.e., $4 \mathrm{~K} /(100 \mathrm{M})=40 \mu$ s. Therefore, the spurious noise suppression can be performed in real time.

The resource occupancy for implementing noise suppression is listed in Tab. 3. As the block RAMs and DSP48E1 slices are the most important resources, they are mainly considered. It is evident that the FPGA is fully competent for the noise suppression from perspective of resource occupancy. 


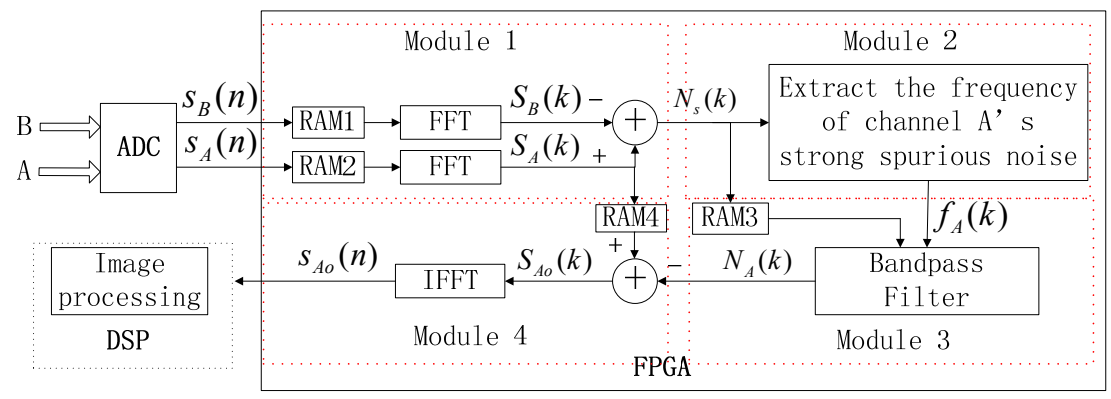

Fig. 5. Block diagram of noise suppression.

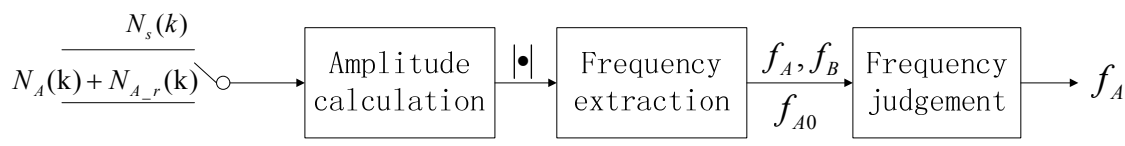

Fig. 6. Extraction flow for the frequency of channel A's strong spurious noise.

\begin{tabular}{|l|c|c|}
\hline & Block RAMs (18 Kb) & DSP48E1 Slices \\
\hline Resource occupancy & 43 & 48 \\
\hline FPGA resource & 312 & 288 \\
\hline Occupancy ratio & $13.78 \%$ & $16.67 \%$ \\
\hline
\end{tabular}

Tab. 3. Resource occupancy for noise suppression.

Considering the precision of result, the data widths of fixed point calculations are all 14 bits. The increased power consumption for the noise of each calculation is $2^{-2 \times 14} /(12 \times 50)=6.2 \times 10^{-12} \mathrm{~W}[10]$. As shown in Fig. 5, there are four series calculation. The total increased power consumption is $6.2 \times 10^{-12} \times 4=2.48 \times 10^{-11} \mathrm{~W}$, which is two order of magnitude lower than the power of ADC strong spurious noise shown in Sec. 3.1.2. Therefore, the precision is high enough.

\subsection{Effectiveness Validation}

To evaluate the effectiveness of the proposed noise suppression method, a simple experiment is performed before the method is used in the image processing. The experiment is now described in detail.

The ADC input is from an external source (model number AWG70000) and the other operations are the same as those in the working order of the radar system. It is noted that the proposed noise suppression method in Section 4.1 is used in the experiment. The input signal is

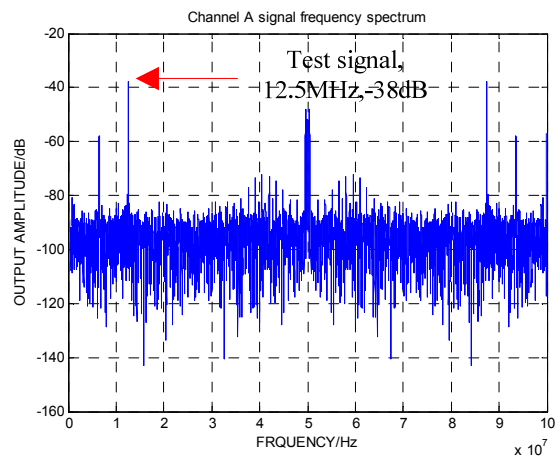

(a)

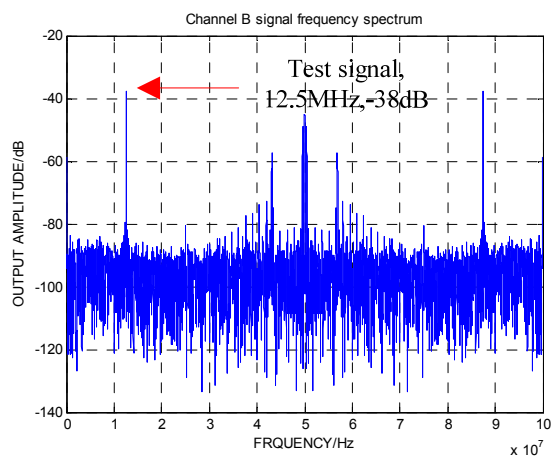

(b)

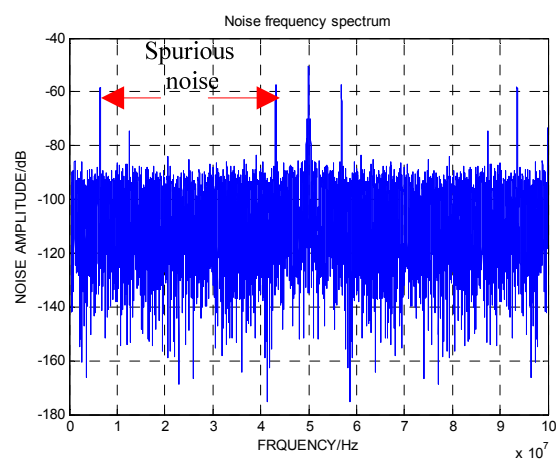

(c)

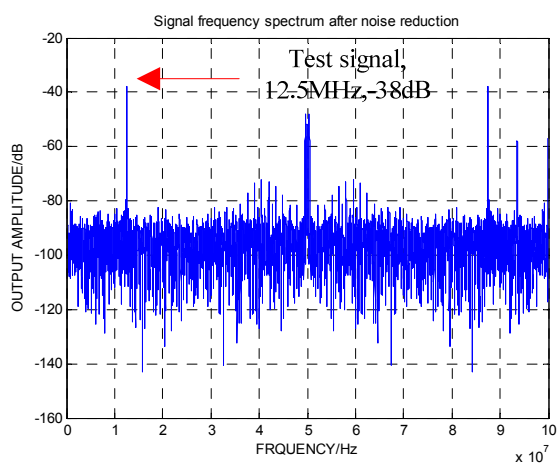

(d)

Fig. 7. Effectiveness validation: (a) Channel A's spectrum; (b) Channel B's spectrum; (c) Subtraction of channel A's spectrum from that of channel B; (d) Spectrum after noise suppression. 
a single-frequency signal with a frequency of $12.5 \mathrm{MHz}$ and amplitude of $-38 \mathrm{~dB}$. The full scale of ADC is $1 \mathrm{~V}$ and is normalized to $0 \mathrm{~dB}$. Figures 7(a) and (b) denote the spectrum of the output signals of channel A and B, respectively. It is shown that the amplitudes of the strong spurious noises of channel $A$ and $B$ are $-57.70 \mathrm{~dB}$ and $-57.80 \mathrm{~dB}$, respectively; the corresponding frequencies are $6.46 \mathrm{MHz}$ and $43.11 \mathrm{MHz}$, respectively. Then the spectrum of channel B's output signal is subtracted from that of channel A's output signal. The amplitude of the result is shown in Fig. 7(c). It is found that there are two strong signals with the same frequency and amplitude as the ADC strong spurious noises in Fig. 7(a) and (b). In addition to the two strong signals, there is a strong signal with a frequency of $12.5 \mathrm{MHz}$ due to the accuracy errors of the resistors that control the amplification of the input signals of channel A and B. Figure 7(d) shows for the spectrum of the signal after noise suppression. There is only a strong signal with the frequency and amplitude of $12.50 \mathrm{MHz}$ and $-38.00 \mathrm{~dB}$, respectively. The amplitude of the spectrum at the $6.46 \mathrm{MHz}$ is $-95.05 \mathrm{~dB}$, which is $37.35 \mathrm{~dB}$ lower than the strong spurious noise. As shown in (21), besides the residual terms $N_{\mathrm{B}}(k)$ after noise suppression there is the term $N_{\mathrm{B}_{\mathrm{r}} \mathrm{r}}(k)$ at the $6.46 \mathrm{MHz}$. Because $N_{\mathrm{B}}(k)$ at the $6.46 \mathrm{MHz}$ is so small that it is difficult to measure the real noise suppression level which is $\left|N_{\mathrm{B}}(k)\right| /\left|N_{\mathrm{A}}(k)\right|$. However, the suppression level of $37.35 \mathrm{~dB}$ has already demonstrated an excellent noise suppression effect in this FMCW SAR.

\section{Experimental Validation}

Figure 8(a) denotes the printed circuit board (PCB) of the signal processor [24]. The noise suppression is performed in the red marked devices and area. An airborne test campaign was performed with the FMCW SAR system. As shown in Fig. 8(b), the FMCW SAR system was mounted on a light airplane [23], [24]. The altitude of the plane is $500 \mathrm{~m}$.

To measure the ISLR, several corner reflectors were placed on an airport. The RCS of corner reflectors, denoted by $R C S_{\mathrm{CR}}$, are approximately to their maximum value of $18.79 \mathrm{dBsm}$. The imaging results with the corner reflectors are shown in Fig. 9(a). The imaging scene is $400 \mathrm{~m} \times 350 \mathrm{~m}$ (range $\times$ Azimuth). Corner reflectors from $\mathrm{T} 1$ to $\mathrm{T} 7$ in Fig. 9(a) are seven point targets. The range and azimuth profiles of corner reflector T2 are shown in Fig. 9(b) and Fig. 9(c), respectively. The peak response of the reflector return is normalized to $0 \mathrm{~dB}$. The average ISLR of seven reflectors are calculated and listed in Tab. 4. The measured $I S L R_{\mathrm{r}}$ in Tab. 4 is approximately to its theoretical value, as shown in Tab. 2. The measured $I S L R_{\mathrm{a}}$ is $-21.76 \mathrm{~dB}$ due to the residual, high-frequency phase errors that were not all eliminated by the motion compensation algorithm. According to [10], a serious deterioration in the imaging results will be caused when the ISLR in azimuth is higher than $-20 \mathrm{~dB}[10]$. Fortunately, the measured $I S L R_{\mathrm{a}}$ of an FMCW SAR is smaller than $-20 \mathrm{~dB}$.

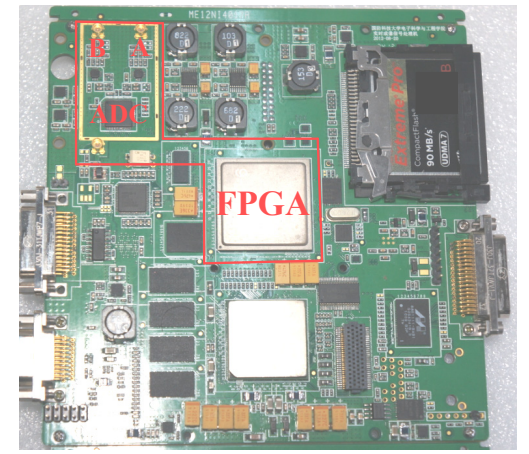

(a)

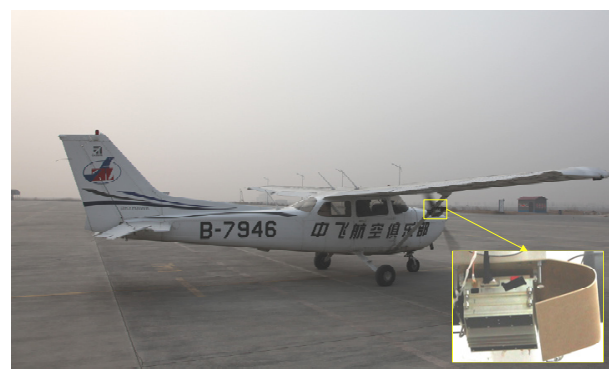

(b)

Fig. 8. Configuration of the test campaign: (a) PCB of the signal processor; (b) SAR system mounted on a light airplane.

\begin{tabular}{|c|c|}
\hline Noise component & Measured result \\
\hline $\boldsymbol{I S L}_{\boldsymbol{r}}$ & $-17.10 \mathrm{~dB}$ \\
\hline $\boldsymbol{I S L}_{\mathbf{a}}$ & $-21.76 \mathrm{~dB}$ \\
\hline ADC strong spurious noise & $-15.83 \mathrm{~dB}(970 \mathrm{~m})$ \\
\hline
\end{tabular}

Tab. 4. Measured results of the noise components.

The value of the receiver thermal noise is sufficiently small, which has marginal influence on the image quality. Therefore, it is not measured in this study.

Measurement of the ADC strong noise uses the SAR data of a quiet lake and the surrounding area where the optical imagery and SAR imagery are shown in Fig. 10(a) and $10(\mathrm{~b})$, respectively. The optical imagery is obtained from Google Earth. The imagery covers areas of $475 \mathrm{~m} \times 620 \mathrm{~m}$ (range $\times$ azimuth) island. It is noted that the proposed noise suppression method is not used when the SAR imagery in Fig. 10(b) was generated. Because the real RCS is required in measuring the noise level, the RCS calibration is performed in Fig. 10. The real signal intensity normalized to $0 \mathrm{~dB}$ in Fig. 10 is equal to that in Fig. 9. The corresponding RCS of the peak response of the reflector' imagery, i.e., $0 \mathrm{~dB}$, is $18.79 \mathrm{dBsm}$.

The sub-image circled by the red rectangle in Fig. 10(b) denotes the imaging results of the scene containing the ADC strong spurious noise. It is shown that a visible curve is present at the range of approximately $970 \mathrm{~m}$, which originates from the ADC strong spurious noise. This curve significantly affects the imagery of the targets, especially that of the low-return areas, such as the lake. The calculation of $\sigma_{\mathrm{sp}}$ is now introduced in detail.

Firstly, the signal intensity of the curve is calculated. The average energy of the curve in the upper lake of 
Fig. $10(\mathrm{~b})$ is denoted by $P_{\mathrm{Ns} r}$. It contains the energy of the ADC strong spurious noise, the water reflection, the additive white noise, and the multiplicative noise. Because the average energy of the last three noise components is approximately equal to that of the surrounding area in the lake, which is denoted by $P_{\mathrm{r}}$. So, the energy of the ADC strong spurious noise is

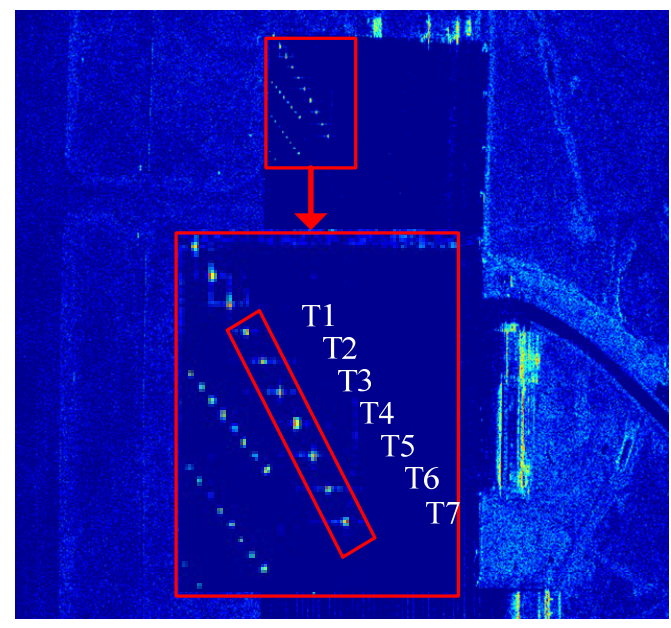

(a)

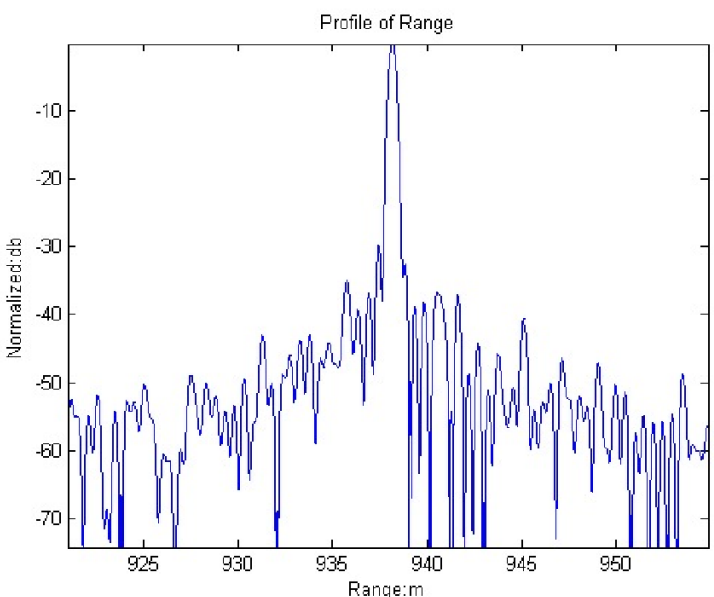

(b)

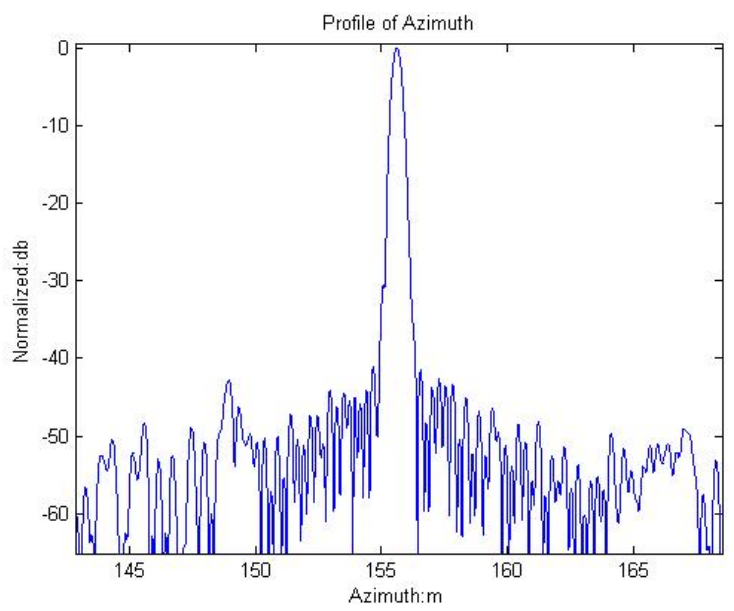

(c)

Fig. 9. Imaging results: (a) Imaging results; (b) Profiles in range [23]; (c) Profiles in azimuth [23]

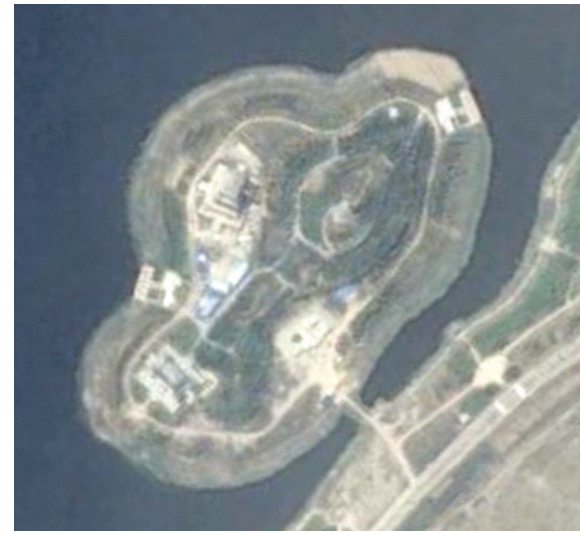

(a)

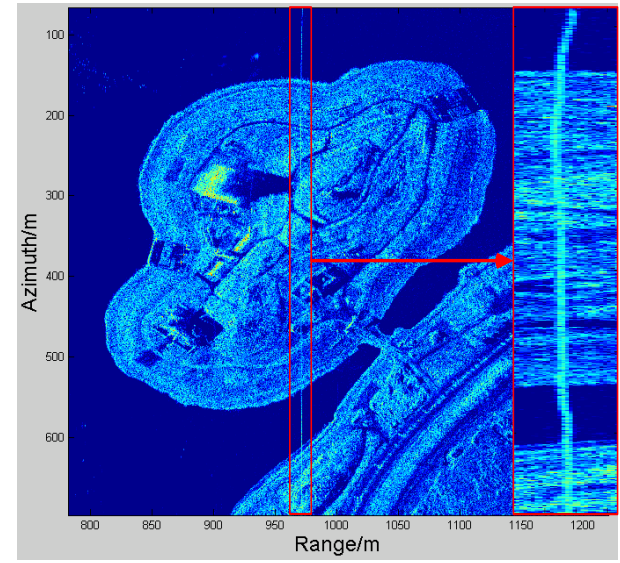

(b)

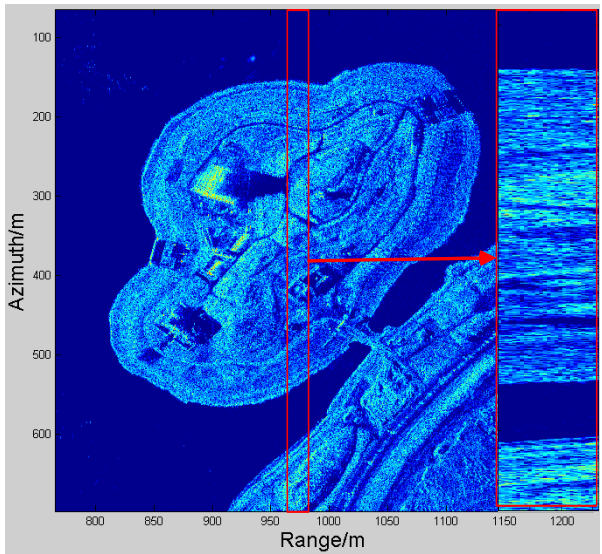

(c)

Fig. 10. Imagery of an island: (a) Optical image. (b) SAR imagery. (d) SAR imagery after noise suppression.

$$
P_{\mathrm{sp}}=P_{\mathrm{r}}-P_{\mathrm{Ns} \_\mathrm{r}} \approx-43.10 \mathrm{~dB} .
$$

The signal energy of the imagery is inversely proportional to $R^{3} \sin \alpha$ [10]. So the energy of the corner reflectors is

$$
P_{\mathrm{CR}}=\mathrm{K} \cdot R C S_{\mathrm{CR}} /\left(R_{\mathrm{CR}}^{3} \sin \alpha_{\mathrm{CR}}\right)
$$

where $K$ is a constant related to the parameters of the FMCW SAR, $R_{\mathrm{CR}}$ and $\alpha_{\mathrm{CR}}$ are the slant range and the pitching angle of the corner reflector, respectively. $R C S_{\mathrm{CR}}$ is the $\mathrm{RCS}$ of the corner reflector, which is [10] 


$$
R C S_{C R}=\sigma_{C R} \rho_{a} \rho_{r} / \sin \alpha_{C R}
$$

where $\sigma_{\mathrm{CR}}$ is the backscatter coefficient of the corner reflector. So

$$
\sigma_{\mathrm{CR}}=R C S_{\mathrm{CR}} \cdot \sin \alpha_{\mathrm{CR}} /\left(\rho_{\mathrm{a}} \rho_{\mathrm{r}}\right) .
$$

Equation (24) can be submitted to (26), the result is

$$
\sigma_{\mathrm{CR}}=P_{\mathrm{CR}} R_{\mathrm{CR}}^{3}\left(\sin \alpha_{\mathrm{CR}}\right)^{2} /\left(K \rho_{\mathrm{a}} \rho_{\mathrm{r}}\right)
$$

Therefore, $\sigma_{\mathrm{sp}}$ is

$$
\sigma_{\mathrm{sp}}=P_{\mathrm{sp}} R_{\mathrm{sp}}{ }^{3}\left(\sin \alpha_{\mathrm{sp}}\right)^{2} /\left(K \rho_{\mathrm{a}} \rho_{\mathrm{r}}\right)
$$

where $R_{\text {sp }}$ and $\alpha_{\text {sp }}$ are the slant range and the pitching angle of the curve. So

$$
\begin{gathered}
\frac{\sigma_{\mathrm{sp}}}{\sigma_{\mathrm{CR}}}=\frac{P_{\mathrm{sp}} R_{\mathrm{sp}}{ }^{3}\left(\sin \alpha_{\mathrm{sp}}\right)^{2}}{P_{\mathrm{CR}} R_{\mathrm{CR}}{ }^{3}\left(\sin \alpha_{\mathrm{CR}}\right)^{2}} \\
\sigma_{\mathrm{sp}} \text { is } \\
\sigma_{\mathrm{sp}}=\frac{P_{\mathrm{sp}} R_{\mathrm{sp}}{ }^{3}\left(\sin \alpha_{\mathrm{sp}}\right)^{2}}{P_{\mathrm{CR}} R_{\mathrm{CR}}{ }^{3}\left(\sin \alpha_{\mathrm{CR}}\right)^{2}} \cdot \sigma_{\mathrm{CR}} \\
=\frac{P_{\mathrm{sp}} R_{\mathrm{sp}}{ }^{3}\left(\sin \alpha_{\mathrm{sp}}\right)^{2}}{P_{\mathrm{CR}} R_{\mathrm{CR}}{ }^{3}\left(\sin \alpha_{\mathrm{CR}}\right)^{2}} R C S_{\mathrm{CR}} \cdot \sin \alpha_{\mathrm{CR}} /\left(\rho_{\mathrm{a}} \rho_{\mathrm{r}}\right) \\
=\left[\begin{array}{l}
10^{-43.10 / 10} \times 10^{18.79 / 10} \\
/\left(1-(500 / 940)^{2}\right) \\
/\left(1-\left(5 S_{\mathrm{CR}}(970 / 940)^{3}\left(1-(500 / 970)^{2}\right)\right.\right.
\end{array}\right. \\
\sqrt{1-(500 / 940)^{2}} /(0.35 \times 0.35) \\
\approx-15.83 \mathrm{~dB} .
\end{gathered}
$$

The theoretical value of $\sigma_{\mathrm{sp}}$ at $970 \mathrm{~m}$ is approximately equal to $-17.32 \mathrm{~dB}$, which is obtained using (12). Therefore, the measured value is approximately $1.51 \mathrm{~dB}$ larger than the theoretical value. There are two probable reasons for this result. There may be manufacturer errors in the triangle reflectors, which could result in a decrease of $R C S_{\mathrm{CR}}$. Or there may be the position errors of the reflectors in the experiment, because the maximum difference in the signal intensity of ten reflectors is $1.40 \mathrm{~dB}$ which is obtained from the data in Fig. 9. Therefore, the real $R C S_{\mathrm{CR}}$ is less than $18.79 \mathrm{dBsm}$.

To evaluate the effectiveness of the proposed method, the proposed noise suppression method is used in another airborne test campaign. The SAR imagery is shown in Fig. 10(c). No curves are shown in the image. However, using other detection methods, the ADC strong spurious noise exist in the scene during the experiment. Therefore, this experimental result shows that the proposed noise suppression method is valid.

\section{Conclusion}

This study is to specify, evaluate and reduce the noise level of a new FMCW SAR system. First, the theoretical derivation of the system's noise level is performed. Then, based on the result of the quantitative analysis, the FFT based noise suppression method is proposed to eliminate the ADC strong spurious noise, adopting an ADC and a FPGA. Finally, using the real Ku-band FMCW SAR data, the levels of the noise components are measured, and the effectiveness of the method of the noise suppression is validated. The results of this study shows that the measured noise levels coincide with the theoretical noise levels, and the proposed method effectively eliminates the ADC strong spurious noise.

It is well known that the sinusoidal interference or NBI is a common problem in different applications. The suppression for this type of interference is oriented towards the application, and the concerns of different applications may vary. Compared with other noise suppression methods, we focus on the noise suppression level at the frequencies of strong spurious noise. The feasibility of our method is validated by experiment, and the performance comparison with other methods shall be arranged as future works.

\section{References}

[1] JiA, G. W., BUCHROITHNER, M., CHANG, W. G., et al. Simplified real-time imaging flow for high-resolution FMCW SAR. IEEE Geoscience and Remote Sensing Letters, 2015, vol. 12, no. 5, p. 973-977. ISSN: 1545-598X. DOI: 10.1109/ LGRS. 2014.2370733

[2] YANG, J. G., HUANG, X. T., JIN, T., et al. Synthetic aperture radar imaging using stepped frequency waveform. IEEE Transactions on Geoscience and Remote Sensing, 2012, vol. 50, no. 5, p. 2026-2036. DOI: 10.1109/ TGRS.2011.2170176

[3] AN, D. X., HUANG, X. T., JIN, T., et al. Extended nonlinear chirp scaling algorithm for high-resolution highly squint SAR data focusing. IEEE Transactions on Geoscience and Remote Sensing, 2012, vol. 50, no. 9, p. 3595-3609. ISSN: 0196-2892. DOI: 10.1109/TGRS.2012.2183606

[4] OTTEN, M., MAAS, N., BOLT, R., et al. Light weight digital array SAR. In Proceedings of the IEEE International Symposium on Phased Array Systems and Technology (ARRAY). Waltham (MA, USA), 2010, p. 177-182. ISBN: 978-1-4244-5127-2. DOI: 10.1109/ARRAY.2010.5613374

[5] EDRICH, M. Design overview and flight test results of the miniaturised sensor MiSAR. In Proceedings of the First European Radar Conference (EURAD). Amsterdam (the Netherlands), 2004, p. 205-208. ISBN: 1-58053-993-9.

[6] NOUVEL, J. F., JEULAND, H., BONIN, G., et al. A Ka band imaging radar: drive on board ONERA motorglider. In Proceedings of the IEEE International Geoscience and Remote Sensing Symposium (IGARSS). Denver (USA), 2006, p. 134-136. ISBN: 0-7803-9510-7. DOI: 10.1109/IGARSS.2006.39

[7] ZAUGG, E. C., HUDSON, D. L., LONG, D. G. The BYU USAR: a small, student-built SAR for UAV operation. In Proceedings of the IEEE International Geoscience and Remote Sensing Symposium (IGARSS). Denver (USA), 2006, p. 411-413. ISBN: 0-7803-9510-7. DOI: 10.1109/IGARSS.2006.110

[8] TIAN, H. S., CHANG, W. G., LI, X. Y. Ambiguity noise analysis of a SAR system. In Proceedings of MIPPR 2015: Multispectral 
Image Acquisition, Processing and Analysis. Enshi (China), 2015, p. 354-358. DOI: $10.1117 / 12.2231541$

[9] CHANG, W. G., JIA, G. W., GU, C. F., TIAN, H. S. Preliminary results of a FMCW SAR with real-time processor. In Proceedings of the International Radar Conference. Hangzhou (China), 2015, p. 1-4. ISBN: $978-1-78561-038-7$. DOI: $10.1049 /$ cp.2015.1487

[10] CARRARA, W. G., GOODMAN, R. S., MAJEWSKI, R. M. Spotlight Synthetic Aperture Radar: Signal Processing Algorithms. 1st ed. London (UK): Artech House, 1995. (Chapter 8) ISBN: 089006-728-7.

[11] SO, H. C. A novel adaptive algorithm for sinusoidal interference cancellation. Electronics Letters, 1997, vol. 33, no. 22, p. 1910 to 1912. ISSN: 0013-5194. DOI: 10.1049/ el:19971295

[12] SO, H. C. Adaptive cancellation of multiple interfering sinusoids Electronics Letters, 1998, vol. 34, no. 24, p. 2301-2302. ISSN: 0013-5194. DOI: 10.1049/el:19981633

[13] VASEghI, S. Advanced Signal Processing and Digital Noise Reduction. 1st ed. Chichester (England): John Wiley \& Sons, 1996. (Chapter 6) ISBN: 978-3-322-92774-3.

[14] NORTH, R. C., ZEIDLER, J. R., ALBERT, T. R., et al. Comparison of adaptive lattice filters to LMS transversal filters for sinusoidal cancellation. In Proceedings of the IEEE International Conference on Acoustics, Speech and Signal Processing. San Francisco (USA), 1992, vol. 4, p. 33-36. ISBN: 0-7803-0532-9. DOI: 10.1109/ICASSP.1992.226418

[15] PFITZNER, M., CHOLEWA, F., PIRSCH, P., et al. A flexible hardware architecture for real-time airborne wavenumber domain SAR processing. In Proceedings of the 9th European Conference on Synthetic Aperture Radar (EUSAR). Nuremberg (Germany), 2012, p. 28-31. ISBN: 978-3-8007-3404-7.

[16] MILSTEIN, L. B., DAS, P. An analysis of a real-time transform domain filtering digital communication system-part I: Narrowband interference rejection. IEEE Transactions on Communications, 1983, vol. 31, no. 1, p. 21-27. ISSN: 0090-6778. DOI: $10.1109 /$ TCOM.1983.1095733

[17] XU, L. J., YAN, Y. Wavelet-based removal of sinusoidal interference from a signal. Measurement Science and Technology, 2004, vol. 15 , no. 9, p. 1779-1786. ISSN: 0957-0233. DOI: $10.1088 / 0957-0233 / 15 / 9 / 015$

[18] ZHAO, Z.Y., CHANG, W.G., LI, X.Y., et al. Range-dependent phase error of dechirp. Journal of National University of Defense Technology, 2014, vol. 36, no. 3, p. 169-176. DOI: 10.11887/j.cn.201403030 (in Chinese)

[19] TAO ZENG, ZHENG LU, DING, Z. G., BIANG, M. M. SAR Doppler ambiguity resolver based on entropy minimization. IEEE Transactions on Geoscience and Remote Sensing, 2013, vol. 51, no. 8, p. 4405-4416. ISSN: 0196-2892. DOI: 10.1109/ TGRS.2013.2240305

[20] ROLT, K. D., SCHMIDT, H. Azimuthal ambiguities in synthetic aperture sonar and synthetic aperture radar imagery. IEEE Journal of Oceanic Engineering, 1992, vol. 17, no. 1, p. 73-79. ISSN: 0364-9059. DOI: $10.1109 / 48.126956$

[21] LIU, M., YU, Z., LI, C. S. Azimuth ambiguity suppression for spaceborne SAR based on PRF micro-variation. In Proceedings of the IEEE International Geoscience and Remote Sensing Symposium IGARSS. Melbourne (Australia), 2013, p. 1325-1328. ISSN: 978-1-4799-1114-1. DOI: 10.1109/IGARSS.2013.6723026
[22] LI, F. K., JOHNSON, W. T. K. Ambiguities in spaceborne synthetic aperture radar systems. IEEE Transactions on Aerospace and Electronic System, 1983, vol. AES-19, no. 3, p. 389-397. ISSN: 0018-9251. DOI: 10.1109/TAES.1983.309319

[23] TIAN, H. S., CHANG, W. G., LI, X. Y. Multiplicative noise analysis of the FMCW SAR. In Proceedings of the International Radar Conference. Hangzhou (China), 2015, p. 1-4. ISBN: 978-178561-038-7. DOI: 10.1049/cp.2015.1485

[24] TIAN, H. S., CHANG, W. G., LI, X. Y. Integrated system of miniSAR real-time signal processing and data storage. In Proceedings of the IEEE Radar Conference. Johannesburg (South Africa), 2015, p. 354-358. DOI: 10.1109/ RadarConf.2015.7411907

[25] XILINX INCORPORATED. LogiCORE IP Fast Fourier Transform V7.1 (datasheet). 63 pages. [Online] Cited 2011-04-19. Available at: http://www.xilinx.com/support/documentation/ ip_documentation/xfft_ds260.pdf.

\section{About the Authors ...}

Haishan TIAN was born in Liaocheng, Shandong Province, P.R. China, in 1987. He received his BS and MSc degree from the National University of Defense Technology (NUDT), Changsha, P.R. China, in 2006 and 2008, respectively. Currently, he is working toward the $\mathrm{PhD}$ degree at the National University of Defense Technology of China. His research interests include signal synthesizer design, radar systems design and SAR signal processing.

Wenge CHANG received the $\mathrm{BS}$, MSc, and $\mathrm{PhD}$ degrees in Information and Communication Engineering from the National University of Defense Technology, Changsha, China, in 1987, 1993, and 2001, respectively. He is currently a professor with the National University of Defense Technology. His current researches include synthetic aperture radar system, and real-time signal processing and SAR image processing.

Xiangyang LI received the $\mathrm{BS}, \mathrm{MSc}$, and $\mathrm{PhD}$ degrees in Information and Communication Engineering from the National University of Defense Technology, Changsha, China, in 1993, 1996, and 2000, respectively. He is currently an associate professor with the National University of Defense Technology. His current researches include signal synthesizer design, synthetic aperture radar systems and signal processing.

Zhaohe LIU received the BS degree in Information and Communication Engineering from the National University of Defense Technology, Changsha, China, in 1987, and the $\mathrm{PhD}$ degree in the Department of Information Technology from PLA University of Science and Technology, Nanjin, China, in 2005 . He is currently a senior engineer with the Science and Technology on Near-Surface Detection Laboratory. His research interests include SAR imaging, mine detection technology. 